\title{
Kolmogorov and Nonstationary Spectra of Optical Turbulence
}

\author{
Vladimir M. Malkin \\ Budker Institute of Nuclear Physics \\ Novosibirsk, 630090, Russia
}

()

\begin{abstract}
Optical turbulence, which develops in the transverse directions when a laser beam propagates through a medium, is considered in the framework of the two-dimensional Schrödinger equation with a weak cubic nonlinearity. This classical model of optical turbulence was studied by many authors, but stationary (Kolmogorov) spectra that transfer transverse energy of the waves from large spatial scales to small ones have not been found until now. Such spectra are given below. Non-stationary optical turbulence which evolution results in formation of a Kolmogorov spectrum is described as well. In addition to this, evolution of free optical turbulence is considered.
\end{abstract}

\section{INTRODUCTION}

Consider nonlinear Schrödinger equation [1]

$$
\left(\imath \frac{\partial}{\partial z}+\Delta+\sigma|\psi|^{2}\right) \psi=0
$$

where $\psi$ is the space-time envelope of electromagnetic field, $z$ is distance along the direction of laser beam propagation, $\Delta$ is the Laplacian in the transverse directions. All the variables are dimensionless. The two cases $\sigma=1$ and $\sigma=-1$ correspond to media with focusing and defocusing nonlinearity, respectively.

Under conditions when the field amplitude is much smaller than the spread of transverse wave numbers in the beam, $\left|\psi_{\text {typ }}\right| \ll k_{\text {typ }}$, the phase differences between typical waves vary much faster than their amplitudes, which leads to statistical self-averaging of the field. Statistical characteristics of such a field may be expressed in terms of its pair correlation function $n\left(\vec{r}_{1}, \vec{r}_{2}, z\right) \equiv\left\langle\psi^{*}\left(\vec{r}_{1}, z\right) \psi\left(\vec{r}_{2}, z\right)\right\rangle$. The angle brackets here signify averaging over the time small in comparison with the time of wave amplitudes variation, but large in comparison with the time of the phase spread variation for $2 \pi$. If both the medium and the turbulence are uniform in a transverse plane $z=$ const, then the pair correlation function is invariant with respect to translations in this plane, and $n\left(\vec{r}_{1}, \vec{r}_{2}, z\right)=n\left(\vec{r}_{1}-\vec{r}_{2}, 0, z\right)$. The Fourier-transformed function, $n_{\vec{k}} \equiv \int d \vec{r} n(\vec{r}, 0, z) \exp (-\imath \vec{k} \vec{r})$, satisfy the so-called kinetic equation for waves (see, for instance, [2]) 


$$
\begin{aligned}
\frac{\partial}{\partial z} n_{\vec{k}}=2 \int \frac{d \vec{k}_{1} d \vec{k}_{2} d \vec{k}_{3}}{(2 \pi)^{6}}(2 \pi)^{2} \delta\left(\vec{k}+\vec{k}_{1}-\vec{k}_{2}-\vec{k}_{3}\right) 2 \pi \delta\left(k^{2}+k_{1}^{2}-k_{2}^{2}-k_{3}^{2}\right) \\
\quad \times n_{\vec{k}} n_{\vec{k}_{1}} n_{\vec{k}_{2}} n_{\vec{k}_{3}}\left(\frac{1}{n_{\vec{k}}}+\frac{1}{n_{\vec{k}_{1}}}-\frac{1}{n_{\vec{k}_{2}}}-\frac{1}{n_{\vec{k}_{3}}}\right) .
\end{aligned}
$$

This equation conserves the wave action ("number of quanta"), transverse momentum and "transverse energy" of the waves,

$$
N=\int d \vec{k} n_{\vec{k}}, \quad \vec{P}=\int d \vec{k} \vec{k} n_{\vec{k}}, \quad E=\int d \vec{k} k^{2} n_{\vec{k}}
$$

The $\delta$-functions in the right hand side of (2) correspond to conservation of the transverse momentum and "transverse energy" in each act of four-wave interaction.

As it must be, the kinetic equation has the thermodynamic equilibrium solution $n_{\vec{k}}=$ $\frac{T}{\mu+k^{2}-\vec{k} \vec{v}}$, which coincides with the classical Rayleigh-Jeans distribution. Here $T$ is the "transverse temperature" of the waves, $\mu$ is their "chemical potential" (see, for instance, [3]), and $\vec{v}$ is the average transverse component of the group velocity of the waves. The transverse momentum of waves equals to zero in the coordinate frame moving with the velocity $\vec{v}$. In such a frame the thermodynamic equilibrium solution of (2) reduces to $n_{\vec{k}}=\frac{T}{\mu+k^{2}}$,

Equations (1) and (2) relate to the conservative system of waves. These equations may also be applicable in a "transparency windows" in $\vec{k}$-space, if the waves inside the windows are not sensitive to the outside regions where a pumping and damping take place. The latter assumption is often identified with the Kolmogorov's hypothesis of "locality" of turbulent interactions in $\vec{k}$-space. However, the "locality", understood as the dominance of interactions between turbulent fluctuations having more or less equal spatial scales, is actually stronger assumption, because the "transparency windows" may contain very different spatial scales.

Consider a "transparency window" $k_{0} \ll k \ll k_{M}$. Let $J$ be the "transverse energy" that comes into the "window" at its lower boundary per unit of the "time" $z$. In a "stationary", i.e. $z$-independent state, the same transverse energy must leave the "window" at its upper boundary per unit of the "time" $z$. This means that the stationary state must carry through the "window" the energy flux $J$. In the thermodynamic equilibrium, the flux $J$ equals to zero. Therefore, a stationary solution of (2) with $J \neq 0$ must differ from the Rayleigh-Jeans distribution. A similar consideration is applicable to a stationary solution of (2) with a constant flux $Q \neq 0$ of wave action $N$ through a "transparency window".

The first example of a Kolmogorov spectrum as an explicit stationary (but not equilibrium) solution of a kinetic equation for waves was found in 1966 by V.E. Zakharov [4]. Then, the theory of Kolmogorov spectra of wave turbulence was developed by many researchers. A review of these developments may be found in [5]. Recent progress in studying optical turbulence is reflected in [6]. The main difficulties of the theory of optical turbulence was found there to be as follow.

Consider a wave pumping that is localized in a region $k \sim k_{0}$ and that generates $Q$ quanta and the transverse energy $J$ per unit of time. Let the region $k \sim k_{0}$ be surrounded from its left and right sides by two transparency windows, $k_{m} \ll k \ll k_{0}$ and $k_{M} \gg k \gg k_{0}$. Then, one may expect to have in the left window the stationary state with $J \approx 0$ and flux 
$Q$ directed to small $k$ 's, and to have in the right window the stationary state with $Q \approx 0$ and flux $J$ directed to large $k$ 's. This is because the transverse energy flux associated with the flux $Q$ tends to zero (like $Q k^{2}$ ) when $k \rightarrow 0$, and the number of quanta flux associated with the flux $J$ tends to zero (like $J k^{-2}$ ) when $k \rightarrow \infty$. Meanwhile the standard method of finding Kolmogorov spectra from kinetic equations gives for (2) the opposite direction of flux $Q$ in the left window and zero value of flux $J$ the right window.

The problem with the left transparency window was basically solved in [6]. The authors looked for a solution in the form $n_{\vec{k}}=\frac{T}{\mu+k^{2}+\phi(k)}, \quad \phi(k) \ll \mu+k^{2}$. They found from the linearized over $\phi(k)$ equation (2) that $\phi(k)=a Q T^{-3} k^{4} \ln ^{2}\left(k_{0} / k\right)$ in the region $k^{2} \gg \mu$, which justifies the linearization, as the function $\phi(k)$ decreases at $k \rightarrow 0$ faster than $k^{2}$.

The attempt to solve in a similar manner the problem with the right transparency window fails, because the function $\phi(k)$ found from the linearized equation (2) increases at $k \rightarrow \infty$ faster than $k^{2}$ (see [6]). The authors of [6] considered also the "logarithmic modification of Kolmogorov spectrum". They showed, assuming the locality of wave interaction, that the distribution $n_{\vec{k}}=c k^{-2} \ln ^{1 / 3}\left(k / k_{0}\right)$ might carry non-zero energy flux $J$. However, this flux appeared to be directed to small $k$ and, apart from this, the spectral density of transverse wave energy, $k^{2} n_{\vec{k}}$, increases infinitely at $k \rightarrow \infty$. Therefore, the above distribution was found in [6] not to be relevant.

Thus, the problem of finding Kolmogorov spectrum with a non-zero energy flux remained open. To solve this problem is the purpose of present paper. We also wish to consider nonstationary solutions that describe formation of the Kolmogorov spectrum for the kinetic equation (2). One more goal consists in studying behavior of free optical turbulence.

\section{BASIC EQUATIONS}

As the "transverse energy" of waves is conserved, its spectral density $T_{\vec{k}} \equiv k^{2} n_{\vec{k}}$ must satisfy a continuity equation. For isotropic spectra, such an equation looks like

$$
\frac{\partial}{\partial z} T_{k}=-\frac{1}{k} \frac{\partial}{\partial k} k j_{k},
$$

where $j_{k}$ is the density of transverse energy flux in $\vec{k}$-space. For stationary spectra, the flux $k j_{k} \equiv J_{k}$ does not depend of $k$. For the equilibrium spectrum, the "transverse temperature of waves" $T_{k}$ does not depend of $k$ and $J_{k}=0$.

The attempt to find a stationary power spectrum with $J_{k} \neq 0$ by means of the standard method leads to the equilibrium spectrum for which $J_{k}=0$. This implies that true Kolmogorov spectrum is somehow close to the Rayleigh-Jeans distribution of waves. It is natural to assume that the "temperature" $T_{k}$ slowly varies along the spectrum, and the gradient of this temperature in $\vec{k}$-space causes a flux of "transverse energy" from higher to lower $T_{k}$. The expression for the flux $j_{k}$ in terms of $T_{k}$ has to be of the form

$$
j_{k}=-D(k) \frac{\partial}{\partial k} T_{k} .
$$

Apart from this, the flux $j_{k}$ must be cubic in $T_{k}$, as the equation (4) coincides with (2), which right hand side is cubic in $n_{k}$. The comparison of (4) and (5) with (2) also shows 
that, at fixed $T_{k}$, the "diffusion coefficient" $D(k)$ must be invariant with respect to rescaling $k \rightarrow$ const $k$ in $\vec{k}$-space.

Under the "locality of interactions" hypothesis, the above properties imply that $D(k)=$ $A T_{k}^{2}, A=$ const $>0$. The equation $k A T_{k}^{2} \frac{\partial}{\partial k} T_{k}=-J_{k}=-J=$ const has solution $T_{k}=\left(\frac{-3 J}{A} \ln \frac{k}{k_{0}}\right)^{1 / 3}$ equivalent to that found in [6], when the flux $J$ is negative. This equation has solution at positive $J$ as well, specifically, $T_{k}=\left(\frac{3 J}{A} \ln \frac{k_{M}}{k}\right)^{1 / 3}$. However, substitution of the corresponding spectrum $n_{k}$ in the right hand side of (2) shows that the integral there diverges at small $k$, which contradicts to the "locality of interactions" hypothesis.

The diverged factor, $N(k) \equiv \int_{0}^{k} d k_{1} k_{1} n_{k_{1}} \equiv \int_{0}^{k} \frac{d k_{1}}{k_{1}} T_{k_{1}}$, may be interpreted as the total number of waves inside sphere of radius $k$ in $\vec{k}$-space. Actually this quantity is finite, and it contributes to the diffusion coefficient $D(k)$ as follows $D(k)=A N(k) T_{k}, \quad A=$ const $>0$. This result may be derived rigorously from the kinetic equation (2). The derivation gives the explicit formula for constant $A$,

$$
A=\frac{1}{2 \pi^{2}} \int d \vec{q}_{1} d \vec{q}_{2} \delta\left(\vec{n}-\vec{q}_{1}-\vec{q}_{2}\right) \delta\left(1-q_{1}^{2}-q_{2}^{2}\right)\left(\frac{q_{1}}{q_{2}} \ln q_{1}+\frac{q_{2}}{q_{1}} \ln q_{2}\right)^{2} .
$$

which does not depend on the direction of unit vector $\vec{n}=\vec{k} / k$.

Summarizing the above consideration, we come to the following reduced equations for a spectrum of optical turbulence, $n_{k} \equiv T_{k} / k^{2}$, which temperature $T_{k}$ slowly varies in $\vec{k}$-space:

$$
\frac{\partial}{\partial z} T_{k}=\frac{A}{k} \frac{\partial}{\partial k} k N(k) T_{k} \frac{\partial}{\partial k} T_{k}, \quad T_{k}=k \frac{\partial}{\partial k} N(k)
$$

Formulae (6)-(7) have more general sense. These are applicable regardless to the nature of interactions between long waves, which wave numbers are much smaller than $k$. In particular, (6)-(7) are valid when the long-wave turbulence is strong and, therefore, it may not be described by the kinetic equation (2). This may be shown taking into account that the main contribution to the wave interactions at given $k$ comes from processes in which lengths of three waves are of the order of $k^{-1}$, while other waves are much longer. Such interactions are not "local" in the Kolmogorov's sense. However, if one redefine the "medium" to include in it all waves which are much longer than $k^{-1}$, then the interactions may be treated as "local" three-wave processes. The long waves (included in the redefined medium) do not affect the balance of "transverse" energy and momentum in these processes, but just provide conservation of large "longitudinal" energy and momentum. The reduced equations for quasi-equilibrium spectra governed by such interactions coincide with (6)-(7).

\section{KOLMOGOROV SPECTRA}

A Kolmogorov spectrum with a constant flux $J_{k}=J$ and slowly varying $T_{k}$ satisfies the equations

$$
A N(k) T_{k} \frac{d}{d \ln k} T_{k}=-J, \quad T_{k}=\frac{d}{d \ln k} N(k)
$$


Changing independent variable $\ln k$ for $N \equiv N(k)$, one can find solution of $(8)$ in the right transparency window $k_{0} \ll k \ll k_{M}$ in the form

$$
T_{k}=T(N)=\left(\frac{3 J}{A} \ln \frac{N_{M}}{N}\right)^{1 / 3}, \quad \ln \frac{k_{M}}{k}=\left(\frac{A}{3 J}\right)^{1 / 3} N_{M} \int_{\frac{N}{N_{M}}}^{1} d p\left(\ln \frac{1}{p}\right)^{-1 / 3} .
$$

Constants of integration $k_{M}$ and $N_{M}$ may be interpreted as the upper boundary of the spectrum and the total number of waves, respectively.

Let $N_{0}$ be the number of waves in the region $k \lesssim k_{0}$ of $\vec{k}$-space. Behavior of the Kolmogorov spectrum given by formulae (9) depends on the relation between $N_{0}$ and $N_{M}$. To clarify this dependence, it is useful to consider extreme cases $N_{M} \gg N_{0}$ and $N_{M}-N_{0} \ll N_{0}$.

Consider first the case $N_{M} \gg N_{0}$, when nearly all of the waves are located in the right transparency window $k_{0} \ll k \ll k_{M}$. Then, it follows from the second formula $(9)$, taken at the lower boundary of the right transparency window,

$$
N_{M}=\frac{1}{\Gamma(2 / 3)}\left(\frac{3 J}{A}\right)^{1 / 3} \ln \frac{k_{M}}{k_{0}}
$$

where $\Gamma$ is the Euler function. In the depth of the right transparency window, where $N_{0} \ll$ $N \ll N_{M}$, formulae $(9)$ are reduced to

$$
\frac{N}{N_{M}}\left(\ln \frac{N_{M}}{N}\right)^{-1 / 3} \cong \Gamma(2 / 3) \frac{\ln \left(k / k_{0}\right)}{\ln \left(k_{M} / k_{0}\right)}, \quad T_{k} \cong\left(\frac{3 J}{A} \ln \frac{\ln \left(k_{M} / k_{0}\right)}{\ln \left(k / k_{0}\right.}\right)^{1 / 3} .
$$

For the spectrum which is close to the Rayleigh-Jeans distribution with $T \sim J^{1 / 3}$ and $\mu \lesssim k_{m}^{2}$ in the left transparency window, the condition $N_{M} \gg N_{0}$ is equivalent to

$$
\ln \frac{k_{M}}{k_{0}} \gg \ln \frac{k_{0}}{k_{m}}
$$

Noteworthy, that interaction between waves located in the depth of the right transparency window and those located outside this window is negligible in the considered case. In this sense, the interaction is "local". However, it is not local in the Kolmogorov's sense, as waves of lengths much longer than $k^{-1}$ are involved effectively in the interactions of waves which lengths are of order of $k^{-1}$.

In the case opposite to (12), nearly all of the waves are located in the left transparency window. Then, $N$ varies very little in the right transparency window, and it follows from formulae (9) (or directly from equations (8)) that

$$
T_{k} \cong\left(\frac{2 J}{A N_{0}} \ln \frac{k_{M}}{k}\right)^{1 / 2}
$$

At fixed $N_{0}$, the spectrum in the right transparency window would be proportional to $J^{1 / 2}$, as it must be for a three-wave interaction in the redefine medium which includes fixed waves from the left transparency window. For the purely weak turbulence, $N_{0}$ depends of $J$ like $J^{1 / 3}$, and $T_{k} \propto J^{1 / 3}$ in both the windows (which secures smoothness of the spectrum at $k \sim k_{0}$ regardless to the value of $J$ ). 


\section{FORMATION OF KOLMOGOROV SPECTRA}

Consider formation of the stationary spectrum in the right transparency window. Let $k_{F}(z)$ be the upper boundary of the spectrum. In the region $k \ll k_{F}$, the spectrum is capable of changing much faster than at $k \sim k_{F}$, so that the stationary distribution has time enough to establish. (Note, that the typical length of wave interaction in the stationary spectrum depends of $k$, roughly speaking, like $\left.k^{2}\right)$. At $k_{0} \ll k \ll k_{F}$, one has formulae (9) again, but with $k_{F}$ and $N_{F}$ instead of $k_{M}$ and $N_{M}$ respectively. In the region $N_{F}-N \ll N_{F}$ (and $k \ll k_{F}$ ) it follows (compare to $(24)$ )

$$
T_{k} \cong\left(\frac{2 J}{A N_{F}} \ln \frac{k_{F}}{k}\right)^{1 / 2}, \quad \ln \frac{k_{F}}{k_{0}}=\left(\frac{A}{3 J}\right)^{1 / 3} N_{F} \int_{\frac{N_{0}}{N_{F}}}^{1} d p\left(\ln \frac{1}{p}\right)^{-1 / 3} .
$$

The formula for $T_{k}$ is not applicable quantitatively in the region $k \sim k_{F}$, because the $z$ dependence of the spectrum is essential there, and apart from this, the "temperature" $T_{k}$ is no longer slow-varying function of $k$. At $k \sim k_{F}$, the spectrum depends basically on the ratio $k / k_{F}$, which implies that

$$
T_{h} \cong\left(\frac{2 J}{A N_{F}}\right)^{1 / 2} g\left(k / k_{F}\right)
$$

The function $g(\xi)$ is determined from the kinetic equation (2) and boundary conditions

$$
g(\xi) \rightarrow 0, \quad \text { at } \quad \xi \rightarrow \infty ; \quad g(\xi) \rightarrow\left(\ln \frac{1}{\xi}\right)^{1 / 2}, \quad \text { at } \quad \xi \rightarrow 0
$$

When the function $g(\xi)$ is known, the position of the upper boundary of the spectrum $k_{F}$ is determined from the conservation law for the "transverse energy". It follows

$$
J z=\int_{k_{0}}^{\infty} d k k T_{k} \cong k_{F}^{2}\left(\frac{2 J}{A N_{F}}\right)^{1 / 2} \int_{0}^{\infty} d \xi \xi g(\xi) .
$$

As dependence $N_{F}$ of $k_{F}$ is logarithmic one, $k_{F}$ increases, roughly speaking, like $z^{1 / 2}$.

Noteworthy, that nearly all of the "transverse energy" is located at $k \sim k_{F}$. This region may be treated as a reservoir for the "transverse energy". While the reservoir is filling, the stationary spectrum is established at $k \ll k_{F}$. As $k_{F}$ increases, new areas of $\vec{k}$-space begin collect the "transverse energy" of the waves.

\section{FREE OPTICAL TURBULENCE}

For a completely transparent medium, weak optical turbulence is described by the kinetic equation (2) in the whole $\vec{k}$-space. Provided the spectral density of waves $n_{\vec{k}}$ is known at the boundary of the medium $z=0$, one has the Cauchy problem for the equation (2). Let

the function $n_{\vec{k}}$ at $z=0$ be smooth and localized in the region $k \sim k_{0}$. Then, the number of waves and transverse wave energy may be estimated as follow: 


$$
N \sim k_{0}^{2} n_{k_{0}}, \quad E \sim k_{0}^{2} N
$$

As the distance from the boundary of the medium increases, the spectrum approaches to the Rayleigh-Jeans distribution in a region $k \ll k_{F}$. The boundary of this region moves in $\vec{k}$-space according to the equation

$$
\frac{d k_{F}^{2}}{d z} \sim N T
$$

which follows from (7) at its limit of applicability $k \sim k_{F}$. Parameters of the spectrum are linked with the conserved quantities $N$ and $E$ by estimates

$$
N \sim T \ln \frac{k_{F}^{2}}{\mu}, \quad E \sim T k_{F}^{2} .
$$

Taking into account (20), one can integrate (19) and get at $z \gg z_{0} \sim k_{0}^{2} / N^{2}$

$$
k_{F} \sim k_{0}\left(z / z_{0}\right)^{1 / 4}, \quad T \sim N\left(z_{0} / z\right)^{1 / 2}, \quad \mu \sim k_{F}^{2} \exp \left[-\left(z / z_{0}\right)^{1 / 2}\right] .
$$

The typical "time" $z$ of $k_{F}$ variation is much larger than "time" of wave interaction at $k \ll k_{F}$, and typical "time" of $\mu$ variation is much larger than "time" of wave interaction at $k \sim \mu^{1 / 2}$. This implies that the Rayleigh-Jeans distribution, in fact, has time enough to establish in the whole region $k \ll k_{F}$.

The applicability condition of the kinetic equation (2) is the most restrictive for the long-wave part of the spectrum, $k^{2} \sim \mu$, where it is equivalent to $\mu \gg T$. At first this condition is satisfied, as $k_{0}^{2} \gg N$. However, $\mu$ decreases exponentially and it becomes of order of $T$ at $z \sim z_{1} \sim z_{0} \ln ^{2}\left(k_{0}^{2} / N\right)$.

At $z \gtrsim z_{1}$ the longest waves are strongly nonlinear, those are not described by the kinetic equation (2), and behavior of the spectrum depends essentially on the sign of nonlinearity in (1).

In the case of focusing nonlinearity, wave collapse develops at the long-wave boundary of the spectrum. The collapse quickly transfers waves to a small-scale region of $\vec{k}$-space in the form of thin filaments. Collapse and further behavior of such filaments were studied in many papers (see, for instance, [7]). The interaction between developed filaments and weak turbulence appears to be negligible. Thus, collapse just provides a sink for long waves. Location of such a sink, $k \sim k_{f}$, is determined from the collapse condition, $k_{f}^{2} \sim T$. In order to extend (19) and (20) to the considered case, it is sufficient to replace $\mu$ by $k_{f}^{2}$ and to treat $N$ as a number of waves remained in the region $k \lesssim k_{F}$. Integration of thus modified equations gives

$$
k_{F} \sim\left(E^{2} z \Lambda\right)^{1 / 6}, \quad T \sim\left(\frac{E}{z \Lambda}\right)^{1 / 3}, \quad N \sim \Lambda T, \quad \Lambda \equiv \ln \frac{k_{F}}{k_{f}} \sim \ln \left(E^{1 / 2} z\right) .
$$

In the defocusing case, nonlinear wave dynamics is not yet studied in detail. However, collapse is known to be forbidden, and strongly turbulent component of the spectrum cannot quickly transfer waves to a small-scale region of $\vec{k}$-space. Therefore, it is natural to assume that the number of waves in the region $k \lesssim k_{F}$ is conserved, and their transverse energy is basically located at $k \sim k_{F}$. Then, integration of (19) with constant $N$ and $T$ given by the second of the relations (20) leads to the formula (21) for $k_{F}$ again. 


\section{SUMMARY AND PLANS}

We derived reduced equations (7) for a quasi-equilibrium spectrum of optical turbulence, which temperature slowly varies in $\vec{k}$-space. The simplicity of these equations enabled us to determine analytically the family of Kolmogorov spectra that transfer transverse energy of the waves from large spatial scales to small ones. We also used these equations to describe qualitatively evolution of free optical turbulence.

A more general family of Kolmogorov spectra that transfer flux of the two conserved quantities - a number of waves and a transverse energy - through the same transparency window may be determined as well. This is possible to do combining ideas of present paper with ideas of paper [6] mentioned above.

We plan to study stability of the Kolmogorov spectra of optical turbulence with respect to spatially uniform perturbations, and to extend the study to perturbations that slowly varies in space. The latter perturbations may be described in the framework of geometric optics approximation for waves. Corresponding extension of kinetic equation (2) is wellknown one. We would like to derive from it the extension of reduced equations (7). Apart from the linear stability problem, such an extension will simplify the study of nonlinear structures in $(\vec{k}, \vec{r})$-space.

\section{ACKNOWLEDGMENTS}

This work was performed while visiting the Mathematics Department at UCLA. Research supported in part by the National Science Foundation through grant \# DMS-9306720. I am thankful to Professor Russel Caflisch for the opportunity to use this support and for nice considerations. 


\section{REFERENCES}

[1] R.J. Chiao, E. Garmire and C.H. Townes, Phys. Rev. Lett., 13, 469 (1964); V.I. Talanov, Pis'ma Zh. Eksp. Teor. Fiz., 2, 218 (1965); P.L. Kelley, Phys. Rev. Lett., 15, 1005 (1965).

[2] A.A. Vedenov, in: Reviews of Plasma Physics, M., A. Leontovich, ed., Plenum, Vol.3 (1967); B.B. Kadomtsev, ibid., Vol. 4 (1968); A.A. Galeev and R.Z. Sagdeev, ibid., Vol. $7(1979)$

[3] L.D. Landau and E.M. Lifshitz, Statistical Physics, translated from the Russian by J.B. Sykes and M.J. Kearsley. Oxford ; New York : Pergamon Press, 1980; E.M. Lifshitz and L.P. Pitaevskii, Physical Kinetics, translated from the Russian by J.B. Sykes and R.N. Franklin. Oxford; New York : Pergamon Press, 1981.

[4] V.E. Zakharov and N.N. Filonenko, Dokl. Akad. Nauk SSSR, 170, 1292 (1966).

[5] V.E. Zakharov, V.S. L'vov and G. Falkovich Kolmogorov Spectra of Turbulence, 1. Wave Turbulence, Springer-Verlag, 1992.

[6] S. Dyachenko, A.C. Newell, A. Pushkarev and V.E. Zakharov, Physica D57, 96 (1992).

[7] V.M. Malkin, Physica, D64, 251 (1993). 